\title{
A Physical Constraint on Perceptual Learning: Tactile Spatial Acuity Improves with Training to a Limit Set by Finger Size
}

\author{
Michael Wong, ${ }^{1,2}$ Ryan M. Peters, ${ }^{1}$ and Daniel Goldreich ${ }^{1,2}$ \\ ${ }^{1}$ Department of Psychology, Neuroscience \& Behaviour and ${ }^{2}$ McMaster Integrative Neuroscience Discovery and Study, McMaster University, Hamilton, \\ Ontario L8S 4K1, Canada
}

In touch as in vision, perceptual acuity improves with training to an extent that differs greatly across people; even individuals with similar initial acuity may undergo markedly different improvement with training. What accounts for this variability in perceptual learning? We hypothesized that a simple physical characteristic, fingertip surface area, might constrain tactile learning, because previous research suggests that larger fingers have more widely spaced mechanoreceptors. To test our hypothesis, we trained 10 human participants intensively on a tactile spatial acuity task. During 4 d, participants completed 1900 training trials (38 50-trial blocks) in which they discriminated the orientation of square-wave gratings pressed onto the stationary index or ring finger, with auditory feedback provided to signal correct and incorrect responses. We progressively increased task difficulty by shifting to thinner groove widths whenever participants achieved $\geq 90 \%$ correct block performance. We took optical scans to measure surface area from the distal interphalangeal crease to the tip of the finger. Participants' acuity improved markedly on the trained finger and to a lesser extent on the untrained finger. Crucially, we found that participants' tactile spatial acuity improved toward a theoretical optimum set by their finger size; participants with worse initial performance relative to their finger size improved more with training, and posttraining performance was better correlated than pretraining performance with finger size. These results strongly support the hypothesis that tactile perceptual learning is limited by finger size. We suspect that analogous physical constraints on perceptual learning will be found in other sensory modalities.

\section{Introduction}

For reasons that are not fully understood, the ability to improve acuity through training (perceptual learning) varies markedly among individuals (for reviews, see Sathian, 1998; Fahle, 2005; Seitz and Dinse, 2007). Why do some individuals undergo more perceptual learning than others? This question is of considerable interest within sensory neuroscience for both fundamental and practical reasons. Fundamentally, we wish to understand how the nervous system learns to process sensorineural inputs to achieve accurate perception; practically, we hope to develop perceptuallearning-based therapies to ameliorate sensory deficits (Huang et al., 2008; Ding and Levi, 2011) and strategies to facilitate the acquisition of challenging sensorimotor skills such as Braille reading.

Here we investigated perceptual learning in the sense of touch. When a structured surface contacts the skin, it evokes a spatially varying discharge pattern in the underlying mechanoreceptor population: a neural activity image encoding the surface structure (Johnson, 2001). This neural image is conveyed to the CNS for perceptual processing. Therefore, an

Received Feb. 2, 2013; revised March 31, 2013; accepted April 22, 2013.

Author contributions: M.W., R.M.P., and D.G. designed research; M.W. and R.M.P. performed research; M.W., R.M.P., and D.G. analyzed data; M.W., R.M.P., and D.G. wrote the paper.

This work was supported by an Individual Discovery Grant from the Natural Sciences and Engineering Research Council of Canada. We thank Ashley Beaulieu, Sophia E. Piro, and Phillip Staibano for assistance with data analysis.

Correspondence should be addressed to Daniel Goldreich, Department of Psychology, Neuroscience \& Behaviour, McMaster University, 1280 Main Street West, Hamilton, Ontario L8S 4K1, Canada. E-mail: goldrd@mcmaster.ca.

DOI:10.1523/JNEUROSCI.0514-13.2013

Copyright $\odot 2013$ the authors $\quad 0270-6474 / 13 / 339345-08 \$ 15.00 / 0$ individual's tactile spatial acuity may be determined jointly by the skin's mechanoreceptor density, which sets the fidelity of the peripheral neural image, and the efficiency with which the CNS processes this input into a percept. We hypothesized that with intensive training, central processing would become progressively more efficient and tactile spatial acuity would consequently improve toward the optimum permitted by an individual's mechanoreceptor density, a physical constraint on perceptual learning.

A direct test of this hypothesis would require the measurement of mechanoreceptor density in the living human fingertip. Unfortunately, Merkel cell mechanoreceptors, which convey fine spatial information (Johnson, 2001), cannot be imaged noninvasively in vivo. However, indirect evidence suggests that Merkel cell spacing increases with fingertip surface area (as demonstrated histologically for Meissner's corpuscles; Dillon et al., 2001). Specifically, sweat pores, beneath which Merkel cells tend to cluster (Yamada et al., 1996), are more closely spaced in smaller fingers (Peters et al., 2009). Furthermore, humans with smaller fingers have correspondingly better passive tactile spatial perception (Peters et al., 2009), suggestive of closer receptor spacing. Therefore, we measured fingertip surface area as a proxy for Merkel cell spacing. We reasoned that a hypothetical optimally trained participant with infinite receptor density would be able to discern infinitesimal spatial detail. Thus, we predicted a function relating an individual's optimum spatial resolution to fingertip surface area: a line of slope equal to that reported for untrained participants by Peters et al. (2009), but with $y$-intercept zero. 
We trained participants for $4 \mathrm{~d}$ on a grating orientation task (GOT) (Johnson and Phillips, 1981; Craig, 1999; Goldreich et al., 2009), progressively increasing task difficulty to elicit perceptual learning. As predicted, we found that participants' tactile spatial acuity improved toward the theoretical optimum set by their finger size. Intriguingly, this result was observed on both the trained and an untrained nonadjacent finger, a finding that provides clues to the cerebral locus of tactile learning.

\section{Materials and Methods}

Participants and protocol overview. Ten young adult participants (four men, six women; ages 18.1-30.9 years; median age, 20.8 years) completed the study. These were selected from among 22 total participants tested (12 participants did not meet our performance qualification criterion; see below). All participants indicated that they were free of diabetes, dyslexia, and nervous system disorders. We screened against these conditions because they can adversely affect somatosensory neurons (Hyllienmark et al., 1995) or tactile performance (Grant et al., 1999). In addition, we confirmed that the index and ring fingers of the dominant hand were free of injuries and calluses. Handedness was assessed by a questionnaire (modified from Oldfield, 1971); eight participants were right-hand dominant and two were left-hand dominant. On day 1, we assessed each participant's tactile spatial acuity on the index and ring fingers of the dominant hand. The participant then completed an intensive $4 \mathrm{~d}$ training program using one of these two fingers (days 1-4). Finally, on day 5, we reassessed the participant's tactile spatial acuity on both fingers and optically scanned the fingertips to measure their surface areas. All procedures were reviewed and approved by the McMaster University Research Ethics Board.

Two-interval forced-choice GOT. We conducted all perceptual assessment and training by means of a two-interval forced-choice GOT. The stimulus surfaces were acetal plastic square-wave gratings with parallel grooves and ridges of equal widths ranging from $0.25 \mathrm{~mm}$ to $3.1 \mathrm{~mm}$ in increments of $0.15 \mathrm{~mm}$. We used the Tactile Automated Passive-Finger Stimulator (TAPS) to apply the gratings to the fingertip, as described in detail in Goldreich et al. (2009). Briefly, the participant rested the dominant hand in prone position on a tabletop with the distal finger pad (index or ring) resting over a tunnel in the table. The grating stimuli rose through the tunnel to contact the stationary finger pad for $\sim 1 \mathrm{~s} \mathrm{(50 \textrm {g }}$ contact force, $4 \mathrm{~cm} / \mathrm{s}$ onset velocity). A force sensor on the fingernail detected any trials in which the finger inadvertently moved and such trials were discarded automatically from analysis. In each trial, a grating twice contacted the participant's finger (with a 2 s interstimulus interval), once with the grooves transverse (horizontal) and once with the grooves parallel (vertical) to the proximal-distal axis of the finger (order chosen randomly; Fig. $1 A$, inset). The participant indicated the interval perceived to contain the horizontally aligned grating by pressing one of two buttons with the nontested hand.

Tactile spatial acuity assessment. We measured each participant's tactile spatial acuity on the index and ring fingers of the dominant hand both before (day 1 ) and after (day 5) the training session. We refer to the day 1 measurement as the participant's initial (i.e., pretraining) GOT threshold and the day 5 measurement as the participant's final (i.e., posttraining) GOT threshold. These measurements were made by controlling the TAPS device with a modified Bayesian adaptive $\psi$ algorithm (Kontsevich and Tyler, 1999), as described in detail in Goldreich et al. (2009). Briefly, we modeled the participant's discriminability $\left(d^{\prime}\right)$ as a power function of groove width $(x)$ and the participant's psychometric function (probabil-
B

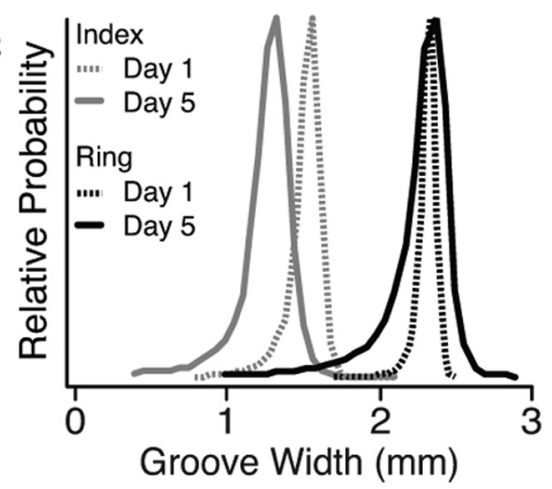

Figure 1. Two-interval forced-choice GOT. A, Participants discriminated the orientation (horizontal, H; vertical, V) of grating stimuli with the stationary index or ring fingertip (inset). Best estimate of the psychometric function of one participant (P3) on days 1 (dashed) and 5 (solid) for the index (gray) and ring (black) fingers. In this participant, training took place on the index finger. The finger. In this participant (contrary to the trend among participants), the psychometric function for the ring finger did not shift, indicating that learning did not transfer to the untrained finger. $\boldsymbol{B}$, Corresponding posterior PDFs for the $76 \%$ correct threshold.

ity correct as a function of $x), \psi(x)$, as a mixture of a cumulative normal curve and a lapse rate term:

$$
d^{\prime}=\left(\frac{x}{a}\right)^{b} \quad \psi(x)=\frac{\delta}{2}+(1-\delta) \frac{1}{\sqrt{2 \pi}} \int^{\frac{d^{\prime}}{\sqrt{2}}} \exp \left(-\frac{y^{2}}{2}\right) d y
$$

The algorithm, which we programmed in LabVIEW for Macintosh (National Instruments) adaptively adjusted groove width from trial to trial to maximize expected information regarding the participant's psychometric function shape parameters: $a$ (position), $b$ (slope), and $\delta$ (lapse rate). We defined the participant's GOT threshold as the $a$ parameter: the groove width at which the participant could correctly discriminate orientation with $76 \%$ probability, corresponding to $d^{\prime}$ equals 1 . The algorithm returned the best-fitting psychometric function (Fig. 1A) as well as a posterior probability density function (PDF) over the $a$ parameter (Fig. $1 B)$.

Testing on each finger began with 20 practice trials with auditory feedback. These were followed by 80 experimental trials without feedback. Participants received 1 min breaks after every 40 experimental trials. The order of finger testing was the same on days 1 and 5 . We initially assigned participants to be tested first on either the index or the ring finger in alternating order upon their entry into the study. We found, however, that only one of five people first tested on the ring finger met our qualification criterion (see below). Accordingly, we modified our protocol to test all subsequent participants first on the index finger. Therefore, of the 10 participants who completed the study, just one (P2) was tested first on the ring finger.

Qualification criterion and training protocol. The intensive training regimen demanded a high degree of sustained concentration from the participants, so we screened participants on day 1 to select those who showed good concentration. When participants concentrate well and perform consistently, the $\psi$ method yields a precise threshold estimate (Goldreich et al., 2009). We therefore assessed concentration by measuring the confidence interval of the day 1 threshold estimates. We initially allowed participants to continue in the study if their index and ring finger $90 \%$ confidence intervals did not span more than five groove widths (i.e., 0.75 $\mathrm{mm}$ ). This criterion, however, proved too stringent: only two of eight participants were able to qualify even after we fixed the order of finger testing (described above). We therefore subsequently relaxed the criterion to apply to the $80 \%$ rather than the $90 \%$ confidence interval.

Participants who met the qualification criterion were trained for $4 \mathrm{~d}$ to discriminate the orientation of grating stimuli. We assigned participants 
in alternating order to be trained on either the index or the ring finger. Immediately after the first testing session, participants completed 8 training blocks of 50 trials each (day 1). They then returned to the laboratory over the next three consecutive days (days 2-4) for further training (10 blocks per day). Therefore, over the $4 \mathrm{~d}$ of training, each participant completed a total of 1900 training trials ( 38 blocks $\times 50$ trials per block). Within a training block, the groove width was held constant. The first training block used the groove width nearest the participant's $76 \%$ correct threshold (mode of the threshold posterior PDF). For each subsequent training block, a groove width was chosen based on the participant's performance on the preceding block: groove width was decreased $0.15 \mathrm{~mm}$ if the participant achieved $\geq 90 \%$ correct, left unchanged if the participant achieved $60-90 \%$ correct, and increased 0.15 $\mathrm{mm}$ if the participant achieved $\leq 60 \%$ correct. Participants received auditory feedback for correct and incorrect responses after each trial. Enforced 1 min breaks occurred between training blocks.

Fingertip surface area measurement. After obtaining the participant's final (posttraining) GOT threshold for both fingers (day 5), we used a flatbed scanner (Epson Perfection 1260) to image the distal volar finger skin at a resolution of $400 \mathrm{dpi}$. The participant placed the hand in prone position on the glass scanner surface; an opaque shield lowered over the hand dorsum exerted gentle downward pressure and protected the participant's eyes from the scanner beam. Using ImageJ software, three observers (Obs) who were blind to the participants' performance separately measured each fingertip's surface area from the distal interphalangeal crease to the tip of the finger; the measurement procedure was identical to that described in Peters et al. (2009). For each fingertip scan, we calculated the percent difference between two observers' surface area measurements as the absolute difference divided by the average measurement. For the index finger, mean interobserver differences were $1.7 \%$ (Obs 1, 2), 1.4\% (Obs 2, 3), and 1\% (Obs 1-3); for the ring finger, mean differences were 1.2\% (Obs 1,2), 1.3\% (Obs 2.3), and 0.7\% (Obs 1-3). Across scans, the observers' measurements were highly correlated (each pairwise correlation between observers for each fingertip: Pearson's $r=$ $0.99, p<0.00001)$. Given this excellent interobserver agreement, we averaged the measurements from the three observers for use in subsequent statistical analysis.

Data analysis. We performed ANOVA, linear regressions, and $t$ tests using SPSS v20 software (IBM) for Macintosh, with an $\alpha$ level of 0.05 ; we report two-tailed $p$-values. The ANOVA model was full-factorial type III sum-of-squares. Unless otherwise stated, the dependent measure was the mean of the posterior PDF over the participant's $76 \%$ correct threshold. Reported correlation coefficients are Pearson's $r$-values.

To assess whether an individual participant's performance on a particular finger improved, we compared the participant's final and initial threshold posterior PDFs, $p\left(a_{\text {final }}\right)$ and $p\left(a_{\text {initial }}\right)$. We calculated the probability $(S)$ that the final threshold was less than the initial threshold as follows:

$$
S=p\left(a_{\text {final }}<a_{\text {initial }}\right)=\int_{a_{\text {final }}=0}^{\infty} p\left(a_{\text {final }}\right)\left(\int_{a_{\text {intial }}=a_{\text {final }}}^{\infty} p\left(a_{\text {initial }}\right) d a_{\text {initial }}\right) d a_{\text {final }}
$$

The $S$ index is a measure of similarity between the two posterior PDFs and has a value between 0 and $1 ; S=0$ if the final threshold PDF is shifted completely (nonoverlapping) to the right of the initial threshold PDF, indicative of a certain worsening in performance; $S=0.5$ if the two PDFs are identical, indicative of no change in performance; and $S=1$ if the final threshold PDF is shifted completely (nonoverlapping) to the left of the initial threshold PDF, indicative of a certain improvement in performance.

In two regression analyses, we required a prediction of the optimal achievable GOT threshold as a function of fingertip surface area. We derived this predicted optimal threshold from a previous GOT study from our laboratory, which tested 97 untrained participants (Peters et al., 2009). In that study, the best-fit line relating GOT threshold ( $y$ ) to finger surface area $(x)$ had a slope of $0.25 \mathrm{~mm}$ threshold increase per square

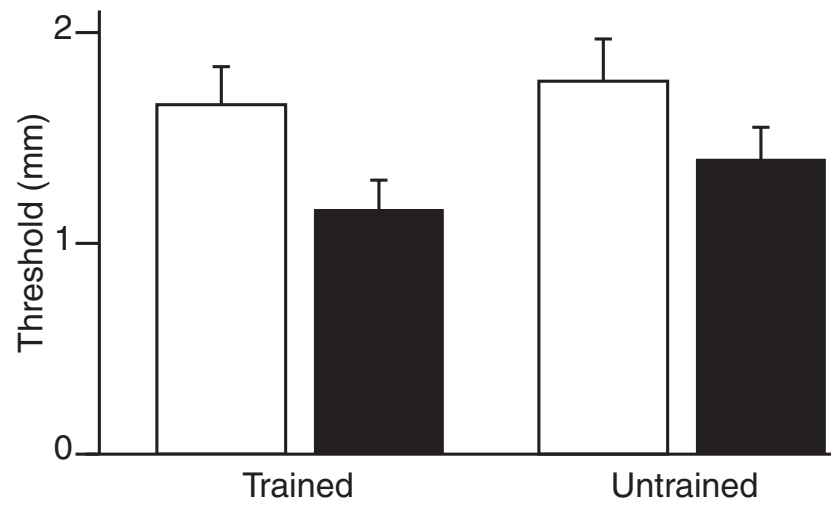

Figure 2. Initial and final thresholds on the trained and untrained fingers. White bars indicate mean initial (day 1) threshold; filled bars, mean final (day 5) threshold. Error bars, 1 SE.

Table 1. S values on the trained and the untrained finger

\begin{tabular}{lll}
\hline & \multicolumn{2}{l}{ Finger } \\
\cline { 2 - 3 } Participant & Trained & Untrained \\
\hline 1 & 0.81 & 0.24 \\
2 & $0.99^{a}$ & $1.00^{a}$ \\
3 & $0.90^{a}$ & 0.47 \\
4 & $1.00^{a}$ & 0.60 \\
5 & $0.97^{a}$ & $0.99^{a}$ \\
6 & $0.96^{a}$ & $0.99^{a}$ \\
7 & $0.91^{a}$ & 0.00 \\
8 & 0.40 & $0.96^{a}$ \\
9 & $1.00^{a}$ & $0.99^{a}$ \\
10 & 0.21 & 0.69 \\
\hline
\end{tabular}

${ }^{a}$ Significant improvement at the 0.9 level.

centimeter of finger surface area, with a $y$-intercept of $0.43 \mathrm{~mm}$. We reasoned that, unlike the untrained threshold, the optimal threshold that would result if participants made full use of the information available from their mechanoreceptors would tend toward zero as the fingertip surface area tended toward zero (and receptor density consequently tended toward infinity). We therefore shifted the best-fit line from Peters et al. (2009) downward to obtain a parallel, predicted optimal threshold line relating finger surface area in square centimeters to threshold, with $y$-intercept zero as follows: $t_{\text {optimal }}=\left(0.25 \mathrm{~mm} / \mathrm{cm}^{2}\right)$ (surface area).

\section{Results}

\section{Spatial acuity improves on both the trained and} untrained finger

To investigate the degree of perceptual learning on the trained and untrained fingers, we conducted a 2 (finger: trained and untrained) $\times 2$ (testing session: initial and final) repeatedmeasures ANOVA, with GOT threshold as the dependent measure. This ANOVA revealed a significant main effect of session $(p=0.030)$, no significant main effect of finger $(p=0.280)$, and no significant finger $\times$ session interaction $(p=0.611)$. The trained finger improved by an average of $0.50 \mathrm{~mm}(\mathrm{SE}=0.19)$ and the untrained finger by an average of $0.37 \mathrm{~mm}(\mathrm{SE}=0.22)$ (Fig. 2).

We next calculated $S$ to assess the degree of improvement of each participant individually on each of the two fingers. With significant improvement defined as $S \geq 0.9$ (and conversely, significant worsening defined as $S \leq 0.1$ ), seven participants improved significantly on the trained finger and five on the untrained finger; one participant worsened significantly on the untrained finger (Table 1). 


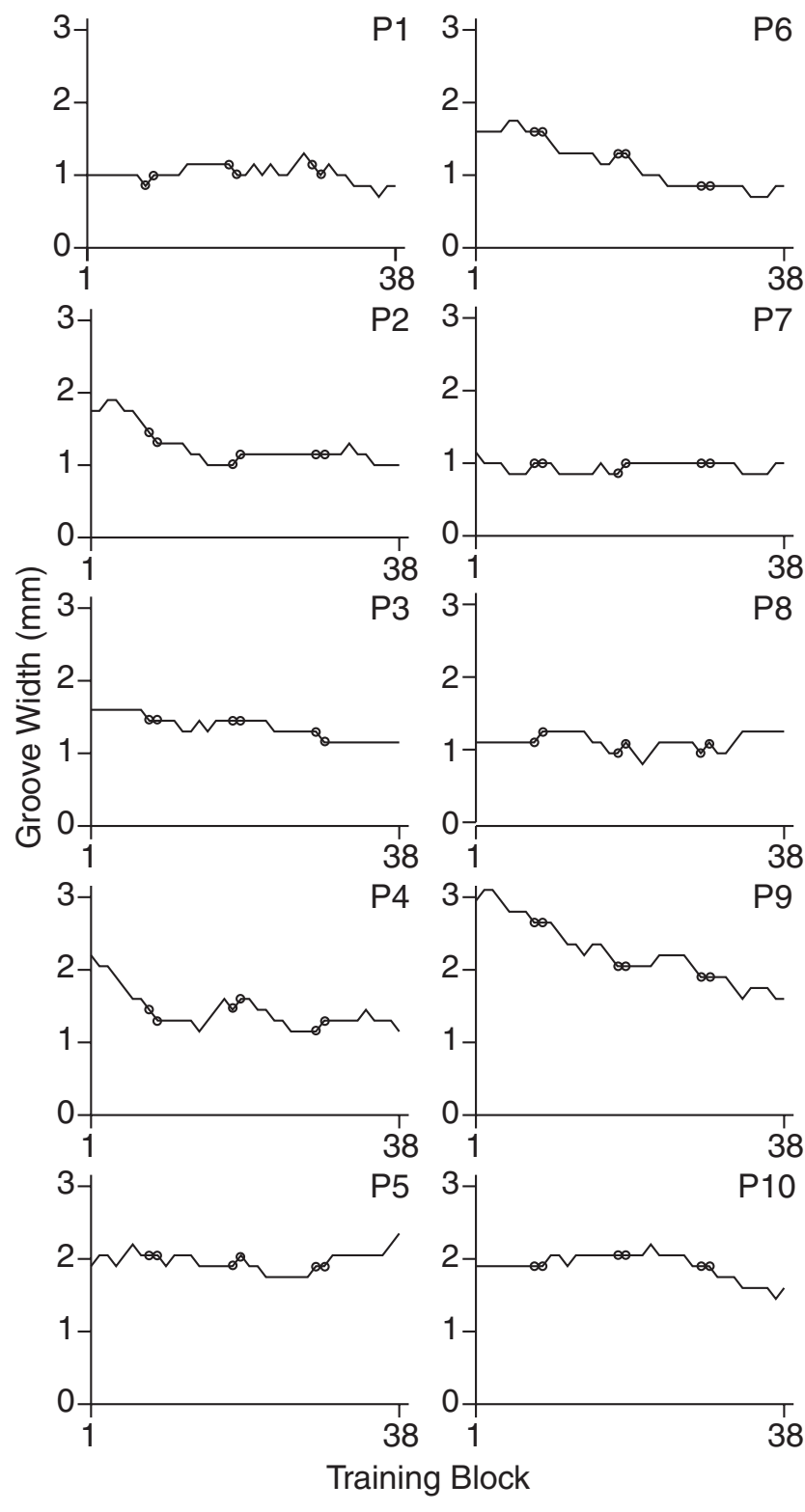

Figure 3. Learning curves (groove width vs training block) for each of the 10 participants (P1-P10). Each participant completed 38 training blocks over the course of $4 \mathrm{~d}$. Adjacent circles indicate the last block on each day and the first block on the subsequent day.

\section{Initial acuity on the trained finger predicts the extent of perceptual learning}

The participants' perceptual learning curves on the trained finger (Fig. 3) showed three salient characteristics. First, perceptual learning appeared to be retained overnight, as the learning curves continued their course without obvious interruption from one day to the next. Second, the learning time course varied markedly from one participant to another; for example, the number of blocks required for participants to improve beyond their initial threshold groove width ranged from two to 30 . Third, those participants with worse initial acuity appeared to undergo more pronounced improvement with training.

Consistent with this last observation, a linear regression revealed that the participants' initial performance predicted their extent of perceptual improvement $(p=0.020, r=0.72$; Fig. $4 A)$. Plausibly, participants with particularly good initial performance improved little with training because their tactile spatial acuity
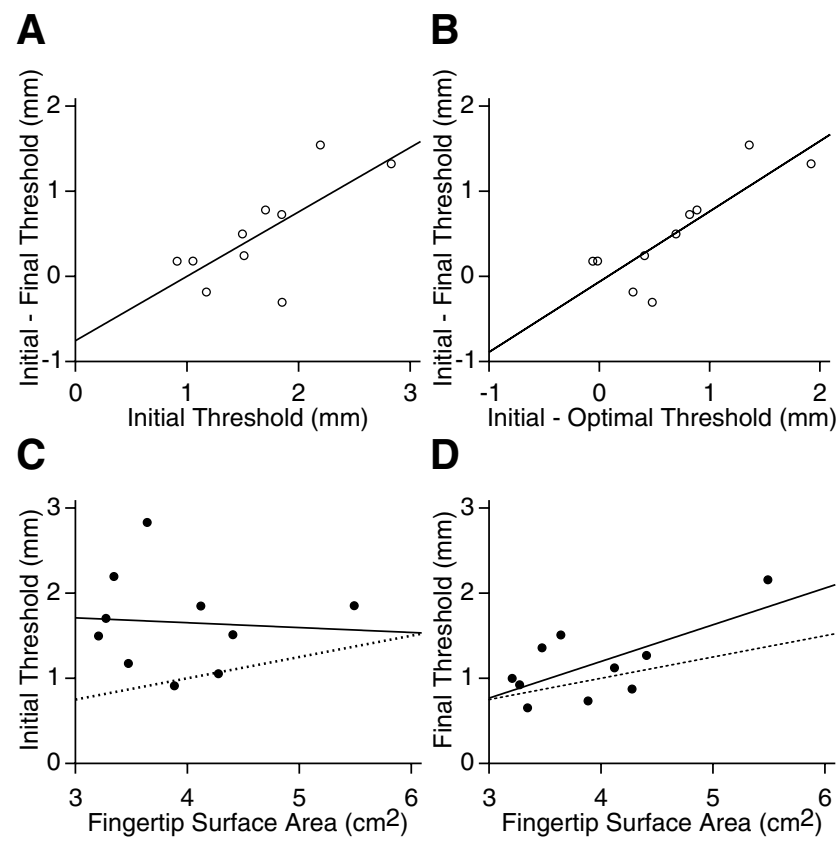

D

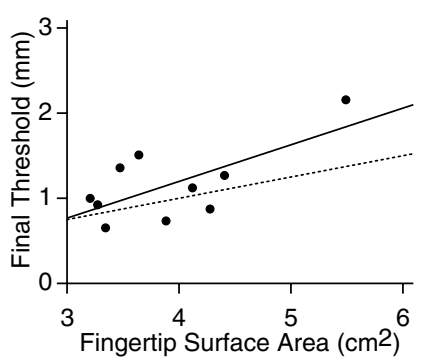

Figure 4. Effects of initial performance and finger size on trained finger performance. $A$, Threshold improvement (initial - final) versus initial threshold. Regression line is shown. B, Threshold improvement (initial - final) versus maximum predicted improvement (initial optimal). Regression line is shown. C, Initial threshold versus fingertip surface area. Solid line is regression line; dotted line is predicted optimal threshold line. $\boldsymbol{D}$, Final threshold versus fingertip surface area. Solid line is regression line; dotted line is predicted optimal threshold line.

was already near its limit; conversely, those with worse initial performance improved more, as they had more room for improvement.

Initial acuity on the trained finger relative to finger size better predicts the extent of perceptual learning

If receptor spacing, as reflected in finger size, determines the optimal limit of spatial acuity, then each participant's maximum potential improvement would equal the difference between the participant's initial threshold and the lowest threshold compatible with the participant's finger size. Therefore, the previous analysis would be made more precise by considering not the initial threshold itself, but the extent to which the initial threshold deviated from the optimal predicted threshold for the participant's finger size. We were able to estimate a relationship between finger size and optimal threshold from the data of a previous GOT study (Peters et al., 2009; see Materials and Methods).

For the trained finger, we plotted each participant's initial threshold deviation from the estimated optimal $\left(t_{\text {initial }}-t_{\text {optimal }}\right)$ against the participant's threshold improvement with training $\left(t_{\text {initial }}-t_{\text {final }}\right)$ (Fig. $\left.4 B\right)$. The deviation of initial from optimal threshold indeed predicted improvement with greater significance and higher correlation (linear regression, $p=0.003, r=$ 0.83 ) than did the initial threshold alone (compare Fig. $4 B, A$ ). The best-fit line (Fig. $4 B$ ) had slope 0.83 (95\% confidence interval: $0.38-1.27)$. Therefore, the participants improved on average by $83 \%$ of the estimated possible improvement given their finger sizes. Finally, we note that the intercept of the best-fit line did not differ significantly from zero (intercept: $-0.06 \mathrm{~mm} ; \mathrm{p}=$ 0.721; $95 \%$ confidence interval: $-0.46-0.33 \mathrm{~mm}$ ), consistent with the logical expectation that, as a participant's potential for improvement $\left(t_{\text {initial }}-t_{\text {optimal }}\right)$ tends to zero, the participant's extent of improvement should also tend toward zero. 


\section{Size of the trained finger predicts spatial acuity after but not before training}

The results of the previous analysis suggested that training drove participants' spatial acuity toward a limit that was determined by their finger size; therefore, we wondered whether GOT thresholds would correlate better with finger size after than before training. Consistent with this prediction, two linear regressions with initial and final thresholds as the dependent variables and finger size as the independent variable revealed that thresholds on the trained finger correlated significantly with finger size after $(p=0.031, r=0.68)$ but not before $(p=0.848, r=-0.07)$ training (Fig. 4C,D).

We note that the initial thresholds of two participants, and the final thresholds of three participants, fell slightly below the optimal threshold line (Fig. 4C,D). However, none of these thresholds was significantly lower than the theoretical optimal. The optimal thresholds (height of the dotted line in Fig. 4C,D) for the five data points were $0.97 \mathrm{~mm}$ and $1.07 \mathrm{~mm}$ (initial threshold points in order of increasing finger size) and $0.84 \mathrm{~mm}, 0.97 \mathrm{~mm}$, and $1.07 \mathrm{~mm}$ (final threshold points in order of increasing finger size). The 95\% confidence intervals for these five data points all crossed the optimal threshold line: the upper bounds of the $95 \%$ confidence intervals were $1.07 \mathrm{~mm}, 1.15 \mathrm{~mm}, 0.98 \mathrm{~mm}, 1.24 \mathrm{~mm}$, and $1.13 \mathrm{~mm}$.

\section{Spatial acuity on the untrained finger shows similar trends with finger size}

Next, we repeated the above analyses, but for the untrained finger. We reasoned that, if tactile acuity is limited by finger size, this limit should apply to any finger. Therefore, performance on the untrained finger, because it improved (Fig. 2), should show similar trends with initial threshold and with finger size as we found for the trained finger.

Consistent with these predictions, we found that the extent of learning on the untrained finger, like that on the trained finger, correlated with initial threshold ( $p=0.013, r=0.75$ ) (Fig. $5 A$ ). The correlation became even sharper and more significant when we used $\left(t_{\text {initial }}-t_{\text {optimal }}\right)$ rather than $t_{\text {initial }}$ alone $(p=0.004, r=$ 0.81 ) (Fig. $5 B$ ). A linear regression on the data shown in Figure $5 B$ revealed a slope of 0.83 (95\% confidence interval: $0.34-1.32$ ); therefore, the participants improved with training on average by $83 \%$ of the estimated possible improvement given their finger sizes. As was the case for the trained finger, the intercept did not differ significantly from zero (intercept: $-0.28 \mathrm{~mm} ; p=0.234$; 95\% confidence interval: -0.78 to $0.22 \mathrm{~mm}$ ). Finally, and again similarly to the results on the trained finger, we found that GOT threshold tended to correlate (although nonsignificantly) better with finger size after $(p=0.154 ; r=0.49)$ than before $(p=0.374$; $r=-0.32$ ) training (Fig. 5C,D).

\section{Comparison with Peters et al. (2009)}

Last, we compared the finger size effect observed in the present study with that observed in 97 untrained participants tested on the index finger by Peters et al. (2009). We reasoned that, because we lowered participants' thresholds toward their limit through training, final thresholds from the present study should on average be lower than those reported by Peters et al. (2009) and they should more tightly correlate with finger size. Both of these predictions were confirmed (Table 2).

\section{Discussion}

In the present study, we found that GOT performance improved on the trained and on a nonadjacent, untrained finger.
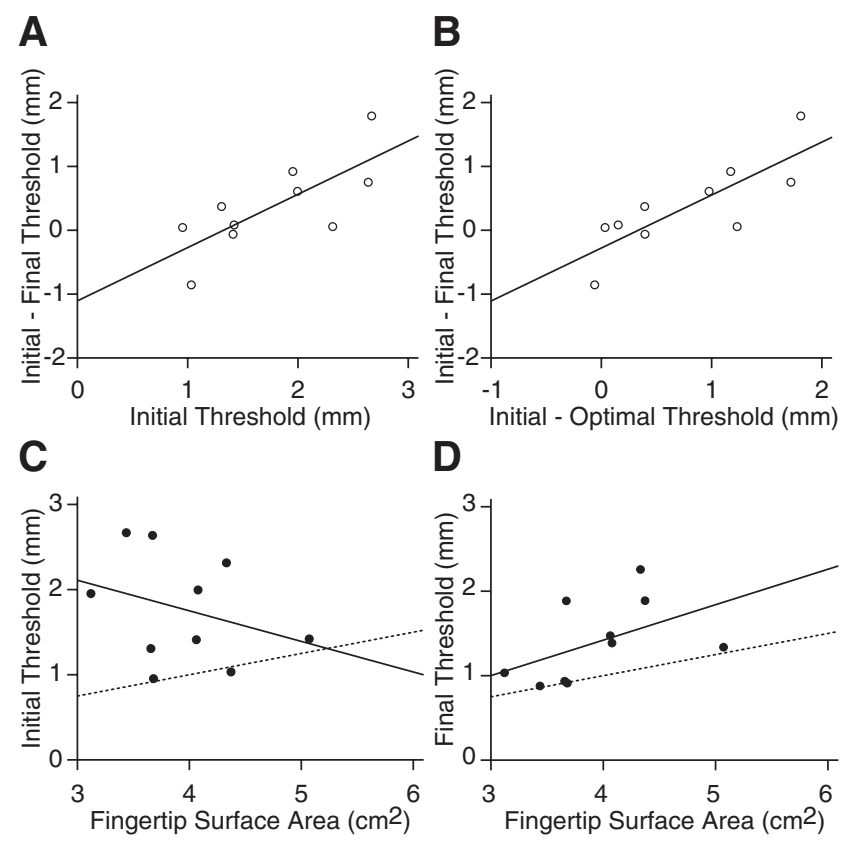

Figure 5. Effects of initial performance and finger size on untrained finger performance. $\boldsymbol{A}$, Threshold improvement (initial - final) versus initial threshold. Regression line is shown. $\boldsymbol{B}$, Threshold improvement (initial - final) versus maximum predicted improvement (initial optimal). Regression line is shown. $\boldsymbol{C}$, Initial threshold versus fingertip surface area. Solid line is regression line; dotted line is predicted optimal threshold line. $\boldsymbol{D}$, Final threshold versus fingertip surface area: Solid line is regression line; dotted line is predicted optimal threshold line.

The extent of improvement was predicted by the difference between a participant's initial performance and the estimated optimal performance for the participant's fingertip size. Furthermore, performance correlated better with fingertip size after than before training. These results support the hypothesis that tactile spatial acuity, and thus the extent of perceptual learning, is limited by finger size.

\section{Tactile perception improves with training}

Training reportedly improves the tactile discrimination of frequency (Harris et al., 2001; Imai et al., 2003), dot location (Sathian and Zangaladze, 1998; Grant et al., 2000; Kauffman et al., 2002), roughness (Sathian and Zangaladze, 1997; Harris et al., 2001), punctate pressure (Harris et al., 2001), and electrotactile patterns (Weiss et al., 2007). In contrast, several studies have reported that GOT performance improves little, if at all, with repeated testing (Van Boven and Johnson, 1994; Goldreich and Kanics, 2003; Wong et al., 2011b; but see Sathian and Zangaladze, 1997).

GOT improvement may have occurred in the present study because: (1) we selected participants for their ability to concentrate well on the task, (2) we gave participants many more training trials than did previous studies, and (3) we used training blocks of fixed groove width. In some previous studies, groove width was adjusted adaptively from trial to trial (Goldreich and Kanics, 2003; Wong et al., 2011b), a procedure that may reduce learning: the intermixing of stimulus levels lessened learning on a visual contrast-discrimination task (Yu et al., 2004).

\section{Perceptual learning transfers to a nonadjacent untrained finger}

Perceptual learning has been reported to transfer from the trained finger to adjacent untrained fingers on tasks involving 
Table 2. Comparison of present study with Peters et al. (2009)

\begin{tabular}{|c|c|c|c|}
\hline & $\begin{array}{l}\text { Present study, trained } \\
\text { finger final threshold }\end{array}$ & $\begin{array}{l}\text { Present study, untrained } \\
\text { finger final threshold }\end{array}$ & $\begin{array}{l}\text { Peters et al. (2009), untrained } \\
\text { participants, index finger threshold }\end{array}$ \\
\hline Threshold versus surface area, correlation ${ }^{a}$ & $r=0.68$ (Fig. 4D) & $r=0.49$ (Fig. 5D) & $r=0.39$ \\
\hline Surface area-adjusted threshold, mean (SD) ${ }^{b}$ & $1.15 \mathrm{~mm}(0.35 \mathrm{~mm})^{c}$ & $1.38 \mathrm{~mm}(0.43 \mathrm{~mm})$ & $1.51 \mathrm{~mm}(0.46 \mathrm{~mm})$ \\
\hline
\end{tabular}

${ }^{a}$ Fingertip surface area better predicted final thresholds in the present study (higher $r$-values).

${ }^{b}$ To compare the two studies' threshold values, we adjusted each threshold, regressing at $0.25 \mathrm{~mm}$ threshold change per cubic centimeter of surface area, to an equivalent fingertip surface area of $3.86 \mathrm{~cm}{ }^{2}$, the median of all fingers tested in both studies. Final thresholds were lower on average in the present study.

$c_{p}<0.05$ compared with Peters et al. (2009), unpaired $t$ test.

discrimination of punctate pressure (Harris et al., 2001), roughness (Sathian and Zangaladze, 1997; Harris et al., 2001), grating orientation (Sathian and Zangaladze, 1997), and frequency (Imai et al., 2003). It has been unclear, however, whether learning transfers to fingers beyond the adjacent ones. Harris et al. (2001) reported no such transfer after a single training session, but Imai et al. (2003), using an intensive multiday training protocol, reported that frequencydiscrimination learning transferred to all tested untrained fingers on both hands. Consistent with Imai et al. (2003), we found with an intensive training protocol that learning transferred to a nonadjacent untrained finger.

\section{Tactile spatial acuity improves with training toward a limit set by finger size}

We found that participants with worse initial performance underwent greater perceptual learning, as reported previously in a visual study (Fahle and Henke-Fahle, 1996). In a tactile study, Sathian and Zangaladze (1998) obtained a similar result, although this was not reported. We performed a linear regression on the data shown in their Table 1 (as in our Fig. 4A), and found that the extent to which participants improved on that study's dot location-discrimination task indeed correlated with their initial performance ( $p=0.009, r=0.77)$. Interestingly, this correlation coefficient is similar to the ones we observed (trained finger, $r=$ 0.72 ; untrained finger, $r=0.75$ ). Therefore, in both studies, initial performance accounted for $>50 \%$ of the explained variance $\left(r^{2}\right)$ in participants' improvement.

We found an even better correlation to GOT improvement when we considered not the initial performance alone, but the deviation between initial performance and the optimal estimated for the finger's size (trained finger, $r=0.835$; untrained finger, $r=0.810)$. The quantity $\left(t_{\text {initial }}-t_{\text {optimal }}\right)$ explained $70 \%$ of the variance in participants' improvement on the trained finger and $66 \%$ on the untrained finger. These results support the hypothesis that tactile spatial acuity is limited by finger size. Consistent with this interpretation, we found that, after training, when the participants' performance was closer to its limit, GOT thresholds correlated more closely with finger size.

Previously, we reported that GOT thresholds increased significantly with finger size in a large group $(n=97)$ of untrained participants (Peters et al., 2009). In that study, considerable variability was observed in thresholds even among participants with similar finger sizes. In the present study, we similarly observed considerable variability in initial thresholds, which, not surprisingly given our comparatively small sample $(n=10)$, masked the finger size effect. Nevertheless, we found that with training, the finger size effect emerged as participants' performance approached its limit and threshold variability diminished (Fig. $4 C, D$, Fig. $5 C, D$ ). Indeed, consistent with this interpretation, finger size better predicted final thresholds in the present study than it did the thresholds of untrained participants in Peters et al. (2009) (Table 2).
A parsimonious explanation for these findings is that Merkel mechanoreceptors, which are responsible for tactile spatial acuity (Johnson, 2001), are more sparsely distributed in larger fingers, as proposed by Peters et al. (2009). Therefore, the lowest achievable threshold (best spatial resolution) for an individual is set by that individual's mechanoreceptor spacing, which is reflected in finger size. We suspect that analogous physical constraints (e.g., photoreceptor density) will be found to explain some of the individual variability in perceptual learning reported in other sensory modalities.

\section{Understanding the sources of individual variation in tactile acuity}

An important goal of tactile research has been to elucidate the sources of the considerable individual variation in tactile acuity. Among the factors discovered to influence tactile spatial acuity are age (Stevens and Patterson, 1995; Goldreich and Kanics, 2003, 2006; Manning and Tremblay, 2006; Wong et al., 2011a) and finger size (Peters et al., 2009). Both factors are thought to correlate with peripheral mechanoreceptor density: receptors are presumably lost with age (Bruce, 1980) and are more dispersed in larger fingers (Dillon et al., 2001). However, even when these factors are accounted for, considerable unexplained variation in spatial acuity remains.

Peters et al. (2009) speculated that, among participants with similar receptor density, another factor, the efficacy of central somatosensory processing, perhaps influenced by daily tactile experience, might account for variability across individuals in tactile spatial acuity. Our finding that tactile training improves spatial acuity supports this hypothesis. We suggest that daily life provides the opportunity for varying degrees of tactile perceptual learning through experience. Therefore, an individual's tactile spatial acuity will reflect not only the individual's age and finger size, but also the individual's degree of everyday reliance on the sense of touch. In the present study, those participants whose initial performance was exceptionally good relative to their finger size may have already driven their spatial acuity toward its limit through daily tactile experience.

There is evidence to suggest that daily somatosensory activities improve tactile spatial acuity. On tactile spatial tasks, pianists outperform nonmusicians (Ragert et al., 2004) and blind individuals outperform the sighted (Stevens et al., 1996; Van Boven et al., 2000; Goldreich and Kanics, 2003, 2006; Legge et al, 2008; Norman and Bartholomew, 2011; Wong et al., 2011a). We reported in a GOT study that blind participants outperformed their sighted peers on the fingers, which blind people rely upon more than the sighted, but not on the lips, where daily experience is presumably similar in the two groups. Furthermore, among blind Braille readers, spatial acuity on the reading fingers correlated with weekly reading experience (Wong et al., 2011a). 


\section{By what neural mechanism does perceptual acuity improve with training?}

As it undergoes perceptual learning, the CNS becomes more efficient at processing afferent sensory input (for a discussion of efficiency within the context of ideal observer analysis, see Geisler, 2003). At an information processing level, this may involve an enhancement of central signal strength (Gold et al., 1999), a reduction in noise (Dosher and Lu, 1998), or both. It is unclear how such improvements in information processing are implemented neurally.

Tactile learning may result from expansions of somatosensory cortical representations (Jenkins et al., 1990; Recanzone et al., 1992; Pascual-Leone and Torres, 1993; Elbert et al., 1995; Xerri et al., 1996, 1999; Sterr et al., 1998; Hodzic et al., 2004). We note that the transfer of learning to an untrained-indeed, nonadjacentfinger (Fig. 2) suggests plastic changes downstream of somatosensory cortical area $3 \mathrm{~b}$, because most area $3 \mathrm{~b}$ neurons have single-finger fields (Sur et al., 1985).

Tactile learning may engage top-down feedback connections that modulate activity within anterior and posterior parietal areas implicated in the GOT (Zhang et al., 2005; Gilbert and Sigman, 2007). Intriguingly, resting posterior parietal $\alpha$ rhythm power and event-related desynchronization of sensorimotor $\alpha$ rhythm predicted the extent of tactile acuity improvement resulting from passive fingertip stimulation (Freyer et al., 2013).

Tactile learning may result from plasticity within perceptual decision networks rather than cortical sensory areas (Sathian et al., 2013). Indeed, the individual variability in learning time course (Fig. 3) raises the intriguing possibility that learning occurs via a stochastic search process in which a decision network selects as inputs a small number of cortical sensory neurons that are most sensitive to the task at hand (Jacobs, 2009).

\section{Notes}

Supplemental material for this article is available at http://psych.momaster. ca/goldreich-lab/Wong_Peters_Goldreich_2013/supplemental.html. This link contains a table listing participant characteristics and a figure depicting the relationship between estimated optimum threshold and fingertip surface area derived from the data of Peters et al. (2009). This material has not been peer reviewed.

\section{References}

Bruce MF (1980) The relation of tactile thresholds to histology in the fingers of elderly people. J Neurol Neurosurg Psychiatry 43:730-734. CrossRef Medline

Craig JC (1999) Grating orientation as a measure of tactile spatial acuity. Somatosens Mot Res 16:197-206. CrossRef Medline

Dillon YK, Haynes J, Henneberg M (2001) The relationship of the number of Meissner's corpuscles to dermatoglyphic characters and finger size. J Anat 199:577-584. CrossRef Medline

Ding J, Levi DM (2011) Recovery of stereopsis through perceptual learning in human adults with abnormal binocular vision. Proc Natl Acad Sci U S A 108:E733-E741. CrossRef Medline

Dosher BA, Lu ZL (1998) Perceptual learning reflects external noise filtering and internal noise reduction through channel reweighting. Proc Natl Acad Sci U S A 95:13988-13993. CrossRef Medline

Elbert T, Pantev C, Wienbruch C, Rockstroh B, Taub E (1995) Increased cortical representation of the fingers of the left hand in string players. Science 270:305-307. CrossRef Medline

Fahle M (2005) Perceptual learning: specificity versus generalization. Curr Opin Neurobiol 15:154-160. CrossRef Medline

Fahle M, Henke-Fahle S (1996) Interobserver variance in perceptual performance and learning. Invest Ophthalmol Vis Sci 37:869-877. Medline

Freyer F, Becker R, Dinse HR, Ritter P (2013) State-dependent perceptual learning. J Neurosci 33:2900-2907. CrossRef Medline

Geisler WS (2003) Ideal observer analysis. In: The visual neurosciences (Chalupa LM, Werner JS, eds), pp 825-838. Cambridge: MIT.
Gilbert CD, Sigman M (2007) Brain states: top-down influences in sensory processing. Neuron 54:677-696. CrossRef Medline

Gold J, Bennett PJ, Sekuler AB (1999) Signal but not noise changes with perceptual learning. Nature 402:176-178. CrossRef Medline

Goldreich D, Kanics IM (2003) Tactile acuity is enhanced in blindness. J Neurosci 23:3439-3445. Medline

Goldreich D, Kanics IM (2006) Performance of blind and sighted humans on a tactile grating detection task. Percept Psychophys 68:1363-1371. CrossRef Medline

Goldreich D, Wong M, Peters RM, Kanics IM (2009) A Tactile Automated Passive-Finger Stimulator (TAPS). J Vis Exp 28:pii 1374. CrossRef Medline

Grant AC, Zangaladze A, Thiagarajah MC, Sathian K (1999) Tactile perception in developmental dyslexia: a psychophysical study using gratings. Neuropsychologia 37:1201-1211. CrossRef Medline

Grant AC, Thiagarajah MC, Sathian K (2000) Tactile perception in blind Braille readers: a psychophysical study of acuity and hyperacuity using gratings and dot patterns. Percept Psychophys 62:301-312. CrossRef Medline

Harris JA, Harris IM, Diamond ME (2001) The topography of tactile learning in humans. J Neurosci 21:1056-1061. Medline

Hodzic A, Veit R, Karim AA, Erb M, Godde B (2004) Improvement and decline in tactile discrimination behavior after cortical plasticity induced by passive tactile coactivation. J Neurosci 24:442-446. CrossRef Medline

Huang CB, Zhou Y, Lu ZL (2008) Broad bandwidth of perceptual learning in the visual system of adults with anisometropic amblyopia. Proc Natl Acad Sci U S A 105:4068-4073. CrossRef Medline

Hyllienmark L, Brismar T, Ludvigsson J (1995) Subclinical nerve dysfunction in children and adolescents with IDDM. Diabetologia 38:685-692. CrossRef Medline

Imai T, Kamping S, Breitenstein C, Pantev C, Lütkenhöner B, Knecht S (2003) Learning of tactile frequency discrimination in humans. Hum Brain Map 18:260-271. CrossRef Medline

Jacobs RA (2009) Adaptive precision pooling of model neuron activities predicts the efficiency of human visual learning. J Vis 9:1-15. CrossRef Medline

Jenkins WM, Merzenich MM, Ochs MT, Allard T, Guíc-Robles E (1990) Functional reorganization of primary somatosensory cortex in adult owl monkeys after behaviorally controlled tactile stimulation. J Neurophysiol 63:82-104. Medline

Johnson KO (2001) The roles and functions of cutaneous mechanoreceptors. Curr Opin Neurobiol 11:455-461. CrossRef Medline

Johnson KO, Phillips JR (1981) Tactile spatial resolution. I. Two-point discrimination, gap detection, grating resolution, and letter recognition. J Neurophysiol 46:1177-1192. Medline

Kauffman T, Théoret H, Pascual-Leone A (2002) Braille character discrimination in blindfolded human subjects. Neuroreport 13:571-574. CrossRef Medline

Kontsevich LL, Tyler CW (1999) Bayesian adaptive estimation of psychometric slope and threshold. Vision Res 39:2729-2737. CrossRef Medline

Legge GE, Madison C, Vaughn BN, Cheong AM, Miller JC (2008) Retention of high tactile acuity throughout the life span in blindness. Percept Psychophys 70:1471-1488. CrossRef Medline

Manning H, Tremblay F (2006) Age differences in tactile pattern recognition at the fingertip. Somatosens Mot Res 23:147-155. CrossRef Medline

Norman JF, Bartholomew AN (2011) Blindness enhances tactile acuity and haptic 3-D shape discrimination. Atten Percept Psychophys 73:23232331. CrossRef Medline

Oldfield RC (1971) The assessment and analysis of handedness: The Edinburgh inventory. Neuropsychologia 9:97-113. CrossRef Medline

Pascual-Leone A, Torres F (1993) Plasticity of the sensorimotor cortex representation of the reading finger in Braille readers. Brain 116:39-52. CrossRef Medline

Peters RM, Hackeman E, Goldreich D (2009) Diminutive digits discern delicate details: Fingertip size and the sex difference in tactile spatial acuity. J Neurosci 29:15756-15761. CrossRef Medline

Ragert P, Schmidt A, Altenmüller E, Dinse HR (2004) Superior tactile performance and learning in professional pianists: evidence for metaplasticity in musicians. Eur J Neurosci 19:473-478. CrossRef Medline

Recanzone GH, Merzenich MM, Jenkins WM, Grajski KA, Dinse HR (1992) Topographic reorganization of the hand representation in cortical area $3 \mathrm{~b}$ 
owl monkeys trained in a frequency-discrimination task. J Neurophysiol 67:1031-1056. Medline

Sathian K (1998) Perceptual learning. Curr Sci 75:451-457.

Sathian K, Zangaladze A (1997) Tactile learning is task specific but transfers between fingers. Percept Psychophys 59:119-128. CrossRef Medline

Sathian K, Zangaladze A (1998) Perceptual learning in tactile hyperacuity: Complete intermanual transfer but limited retention. Exp Brain Res 118: 131-134. CrossRef Medline

Sathian K, Deshpande G, Stilla R (2013) Neural changes with tactile learning reflect decision-level reweighting of perceptual readout. J Neurosci 33: 5387-5398. CrossRef Medline

Seitz AR, Dinse HR (2007) A common framework for perceptual learning. Curr Opin Neurobiol 17:148-153. CrossRef Medline

Sterr A, Müller MM, Elbert T, Rockstroh B, Pantev C, Taub E (1998) Perceptual correlates of changes in cortical representation of fingers in blind multifinger Braille readers. J Neurosci 18:4417-4423. Medline

Stevens JC, Patterson MQ (1995) Dimensions of spatial acuity in the touch sense: changes over the life span. Somatosens Mot Res 12:29-47. CrossRef Medline

Stevens JC, Foulke E, Patterson MQ (1996) Tactile acuity, aging, and Braille in long-term blindness. J Exp Psychol Appl 2:91-106. CrossRef

Sur M, Garraghty PE, Bruce CJ (1985) Somatosensory cortex in macaque monkeys: laminar differences in receptive field size in areas $3 \mathrm{~b}$ and 1. Brain Res 342:391-395. CrossRef Medline

Van Boven RW, Johnson KO (1994) The limit of tactile spatial resolution in humans: Grating orientation discrimination at the lip, tongue, and finger. Neurology 44:2361-2366. CrossRef Medline
Van Boven RW, Hamilton RH, Kauffman T, Keenan JP, Pascual-Leone A (2000) Tactile spatial resolution in blind Braille readers. Neurology 54: 2230-2236. CrossRef Medline

Weiss T, Walter K, Spohn D, Richter M, Torma F, Miltner WH (2007) Spatial discrimination learning of electrocutaneous stimuli. Neurosci Lett 427:83-87. CrossRef Medline

Wong M, Gnanakumaran V, Goldreich D (2011a) Tactile spatial acuity enhancement in blindness: Evidence for experience-dependent mechanisms. J Neurosci 31:7028-7037. CrossRef Medline

Wong M, Hackeman E, Hurd C, Goldreich D (2011b) Short-term visual deprivation does not enhance passive tactile spatial acuity. PLoS One 6:e25277. CrossRef Medline

Xerri C, Coq JO, Merzenich MM, Jenkins WM (1996) Experience-induced plasticity of cutaneous maps in the primary somatosensory cortex of adult monkeys and rats. J Physiol Paris 90:277-287. CrossRef Medline

Xerri C, Merzenich MM, Jenkins W, Santucci S (1999) Representational plasticity in cortical area $3 \mathrm{~b}$ paralleling tactual-motor skill acquisition in adult monkeys. Cereb Cortex 9:264-276. CrossRef Medline

Yamada N, Kashima Y, Inoué T (1996) Scanning electron microscopy of the basal surface of the epidermis of human digits. Acta Anatomica 155:242248. CrossRef Medline

Yu C, Klein SA, Levi DM (2004) Perceptual learning in contrast discrimination and the (minimal) role of context. J Vis 4:169-182. CrossRef Medline

Zhang M, Mariola E, Stilla R, Stoesz M, Mao H, Hu X, Sathian K (2005) Tactile discrimination of grating orientation: fMRI activation patterns. Hum Brain Mapp 25:370-377. CrossRef Medline 